\title{
Immunogenicity of intraperitoneal insulin infusion using programmable implantable devices
}

\author{
N. Jeandidier ${ }^{1}$, S. Boivin ${ }^{1}$, R.Sapin ${ }^{2}$, F.Rosart-Ortega ${ }^{1}$, B. Uring-Lambert ${ }^{3}$ Ph. Réville $^{1}$, M. Pinget ${ }^{1}$ \\ ${ }^{1}$ Service d'Endocrinologie et des Maladies de la Nutrition, Hôpitaux Universitaires, Hôpital Civil, Strasbourg, France \\ ${ }^{2}$ Institut de Physique Biologique, Hôpitaux Universitaires, Hôpital Civil, Strasbourg, France \\ ${ }^{3}$ Institut d'Immunologie, Hôpitaux Universitaires, Hôpital Civil, Strasbourg, France
}

Summary Intraperitoneal insulin infusion using implantable devices in insulin-dependent diabetic (IDDM) patients is promising since it improves diabetic control and decreases frequency of hypoglycaemia. However, preliminary data show a striking increase in plasma levels of anti-insulin antibodies with this therapy. In order to more precisely evaluate the immunogenicity and its consequences, anti-insulin antibody levels in 62 IDDM patients were assessed every 3 months during a 2 -year period following pump implantation. At the same time, diabetes control was evaluated with $\mathrm{HbA}_{1 \mathrm{c}}$, mean blood glucose levels, standard deviation of the daily blood glucose levels and the frequency of low blood glucose $(<3.58 \mathrm{mmol} / 1)$. Factors involved in antibody formation such as age, gender, HLA typing, and complement C4 alleles were also studied. After implantation, anti-insulin antibody levels increased significantly from $3.14 \%$ (range $0-26 \%$ ) to $8.34 \%(0-49 \%$ ) after 1 year and remained elevated. Patients were divided into two groups: responders able to show at least one antiinsulin antibody titre higher than $15 \%$ and non-responders whose titres were always lower than $6 \%$. None of the factors studied was shown to statistically influence the anti-insulin antibody titres. Non-responders had significantly better metabolic results than the responders. Severe hypoglycaemic episodes decreased dramatically in both groups. Insulin requirements were comparable at time 0 and decreased initially in both groups. They remained low for the non-responders but returned to pre-implantation values for responders. Intraperitoneal insulin infusion led to a high immunogenetic response towards insulin in about half of the patients, leading to only moderately deleterious effects on metabolic control. Further studies are necessary to document other consequences (such as the role of circulating immune complexes). [Diabetologia (1995) 38: 577-584]

Key words Anti-insulin antibody, insulin-dependent diabetes mellitus, implantable pump, intraperitoneal insulin therapy, diabetes control, hypoglycaemia.
Intraperitoneal (i.p.) insulin infusion using implantable programmable pumps has now been studied in large-scale multicentre trials for more than 3 years. Safety and feasability have already been assessed [1, 2] as well as the patients' acceptability of this techni-

Received: 3 May 1994 and in revised form: 29 September 1994

Corresponding author: Dr. N.Jeandidier, Service d'Endocrinologie et Maladies Metaboliques, HU Strasbourg, I Place de l'hôpital, F-67091, Strasbourg, France

Abbreviations: IDDM, Insulin-dependent diabetes mellitus; AIA, anti-insulin antibodies; MI, multiple injections; CSII, continuous subcutaneous insulin injection. que mainly due to the improvement in quality of life. The first results of efficacy studies are encouraging [3] since they demonstrate a drastic reduction in the incidence of severe hypoglycaemic episodes when compared to subcutaneous insulin administration [2, 4]. However, preliminary data from our group and from others have shown a striking increase in anti-insulin antibody levels (AIA) in plasma during longterm i.p. insulin infusion using implanted devices [59]. The aim of this study was to evaluate more precisely the immunogenicity of this new method of treatment, to define the different factors involved and to assess the eventual clinical and metabolic consequences of this immune reaction. 
Table 1. Pre-implantation characteristics and AIA levels of Group I. Characteristics and AIA of Group II (unselected IDDM patients)

\begin{tabular}{lcc}
\hline & $\begin{array}{l}\text { Group I } \\
(n=62)\end{array}$ & $\begin{array}{l}\text { Group II } \\
(n=62)\end{array}$ \\
\hline Age (years) & $37.00 \pm 1.15$ & $33.08 \pm 1.46$ \\
Diabetes duration (years) & $17.18 \pm 1.12$ & $15.00 \pm 1.39$ \\
Body mass index $\left(\mathrm{kg} / \mathrm{m}^{2}\right)$ & $24.18 \pm 0.34$ & $23.50 \pm 0.45$ \\
Mean ALA levels $(\%)$ & $3.40(0-26)$ & $2.35(0-16)$ \\
\hline
\end{tabular}

Mean \pm SEM for age, diabetes duration and body mass index, and as mean, higher and lower titres for AIA

\section{Subjects and methods}

Patients. Two groups of patients were studied. Group I included 62 patients who were treated with an implantable programmable insulin pump. They were all, C-peptide negative insulindependent diabetic (IDDM) patients with at least 1 year diabetes duration. All had been treated with human insulin at least 3 months before implantation. Exclusion criteria included major medical or psychiatric disorders and severe late diabetic complications such as advanced nephropathy or unstabilized proliferative retinopathy. Group II included 62 unselected IDDM patients treated subcutaneously with human insulin, these patients serving as a control population. At the time of implantation, Group I patients and Group II patients were similar regarding body mass index, age and diabetes duration as shown in Table 1.

Devices and insulin. Three different types of devices were used in this study; Infusaid Model 1000 (Shiley Infusaid, Norwood, Mass., USA), Minimed MIP 2001 (Minimed Technologies, Sylmar, Calif., USA) and Siemens Promedos ID3 (Siemens Elema, Solna, Sweden) [10].

The insulin used in all three devices is the $21 \mathrm{PH}$ human semi-synthetic, Genapol stabilized insulin (Hoechst A.G. Frankfurt, Germany); a concentration of $400 \mathrm{U} / \mathrm{ml}$ for the $\mathrm{MIP}$ and $100 \mathrm{U} / \mathrm{ml}$ for the ID3 and the Infusaid 1000. The Genapol (polyethylenepolypropilene glycol) at a concentration of $10 \mathrm{ug} / \mathrm{ml}$ acts as a surfactant avoiding the deformation of insulin molecules which may lead to insulin degradation (loss of biological activity and aggregates formation).

\section{Methods}

Immunogenicity of i. p. insulin infusion was assessed as follows: Serum was tested for insulin antibodies using the Biomerieux (Lyon, France) radiobinding assay. The determination is performed in two steps according to the manufacturer's protocol. Serum samples $(50 \mu \mathrm{l})$ were incubated for $24 \mathrm{~h}$ at $4{ }^{\circ} \mathrm{C}$ with $200 \mu 1$ of $\mathrm{I}^{125}$ porcine insulin in albumin phosphate buffer.

Following incubation, $30 \mu \mathrm{l}$ of the mixture was incubated with $50 \mu \mathrm{l}$ of normal human serum, $100 \mu \mathrm{l}$ of human total anti$\mathrm{IgG}$ serum and $3 \mathrm{ml}$ of $24 \%$ polyethyleneglycol solution. The precipitate was centrifuged at $2000 \mathrm{~g}$ for $20 \mathrm{~min}$ at $4^{\circ} \mathrm{C}$. The supranatant was decanted and the pellets were assessed for radioactivity in a gamma-counter. The results are expressed as the percentage of bound counts $(100 \times$ bound counts / total input counts). The non-specific binding measured with a normal serum was subtracted from each sample result. Presence of AIA in a serum was established when the binding percentage was higher than $2 \%$.
The intra-assay coefficient of variation was $7.9 \%$ for an antibody level of $8.1 \%, 9$ for $17.3 \%, 7.6$ for $37.9 \%$. The between assay coefficient of variation was 15.3 for $7.9 \%, 16.3$ for $21.3 \%$ and 9.2 for $46.7 \%$.

Diabetes control was assessed by $\mathrm{HbA}_{\mathrm{Ic}}$ measurements which were performed using the DIAMAT HPLC System (BioRad, Hercules, Calif., USA) [11], (normal values from 4.3 to $6.1 \%$ ) and patients' capillary blood glucose measurements were recorded using a memory glucose meter such as the Glucometer M (Bayer-Ames, Miles, USA) or the Glucoscan One Touch II (LifeScan, Johnson and Johnson, Milpitas, Calif., USA).

Genetic background of the patients was assessed by HLA determination. Class II typing was performed on B lymphocytes isolated with magnetic immunobeads according to the method of Vartdal et al. [12]. The C4 complement factor polymorphism was assessed using an electrophoretic technique on the plasma samples previously treated with either neuraminidase alone or carboxypeptidase and neuraminidase.

Insulin requirements were calculated using the volume of insulin actually infused by the pump, corrected by the number of days between two refills.

Study design and protocol. All the patients were initially selected according to a feasibility and safety protocol. The protocol was approved by the local ethical committee. The pumps were implanted under general anaesthetic, in the left lower quadrant of the abdomen, and sutured to the fascia. The catheter was inserted blindly into the peritoneal cavity. Patients returned to the clinic at least every 3 months, for a refill or if any problems occurred.

A memory glucose meter was given to each patient about 1 month prior to implantation. They were told to perform at least four capillary measurements per day and measure postprandial blood glucose levels regularly; these data were entered in the memory as M1 (preprandial) or M2 (postprandial) in order to perform statistical analysis. The Glucofacts Data Management System (Ames) enables the calculation, for 1 month, of mean blood glucose levels, of the mean 24-h blood glucose standard deviations [5] which is a parameter used to evaluate blood glucose excursions and of the number of times blood glucose falls below $3.58 \mathrm{mmol} / \mathrm{l}$.

Biomerieux AIA levels were determined for Group I and Group II patients. In Group I patients, AIA were assessed during the 4 weeks preceding the implantation and then every 3 months. $\mathrm{HbA}_{1 \mathrm{C}}$ levels were measured at the time of implantation and then every 3 months at each AIA assessment. Daily insulin requirements were determined at each refill; therefore, the first evaluation was done 1 or 2 months after implantation, depending on the type of device.

Three types of patients were characterized at the end of the study by assessing the division of the population by ALA levels calculated from 12 to 24 months (Fig. 1 ).

Responders $(n=25)$ whose AIA titres reached at least once a level of $15 \%$, (these patients were considered to have a high antigenic reaction against the insulin used).

Non-responders $(n=24)$ whose AIA titres were always lower than $6 \%$ during the entire study.

Thirteen patients had intermediate values. 


\section{Statistical analysis}

All data are presented as means \pm SEM unless otherwise stated. All AIA data were transformed to logarithms (after the addition of one to each number) for each antibody level in order to normalize the distribution of values before statistical evaluations [5]. All data are expressed as percent and are derived from the logarithms. The mean of 12 to 24 months were compared to the pre-implantation levels using the paired Student's $t$-test of the log-transformed data since it is not relevant to repeat the tests for each period of time. Chi-square was used for all frequency comparisons. A linear regression was used to evaluate the relationship between the pre-implantation AIA levels and the peak reached in the post-implantation period.

Statistical significance was considered for $p$-values less than 0.05 .

\section{Results}

AIA levels in Group I prior to implantation; comparison with Group II. Prior to implantation, the AIA levels in Group I (mean $=3.40$, range: 0 to $26 \%$ ) were not significantly different from the AIA levels found in Group II patients (mean $=2.35 \%$, range: 0 to $16 \%)$. Of the patients $6.5 \%(n=4)$ in Group I prior to implantation and $1.6 \%(n=1)$ in Group II had an AIA level higher than $15 \%$; at the same time $82 \%$ $(n=51)$ of patients in Group I and $84 \%(n=52)$ in Group II had AIA levels lower than $6 \%$.

Evolution of AIA levels during i.p. insulin infusion in Group I. After implantation, AIA levels in Group I increased significantly from $3.40 \%(0-26 \%)$ at month 0 to $8.34 \%(0-49 \%$ ) (mean of month 12 to month 24) (Fig. 2). The percentage of patients whose AIA was equal or higher than $15 \%$ increased from $6.5 \%(n=4)$ at months 0 to $40 \%(n=29)$ after 1 year, and remained stable thereafter. Mean AIA titres in the responder group increased after 3 months compared to the pre-implantation period and remained elevated thereafter.

\section{Factors involved in AIA formation}

Age: Mean age at the time of implantation was comparable for responders $(37.84 \pm 2.04$ years $)$ and nonresponders $(35.29 \pm 1.80$ years $)$. Of the six patients over 50 years old, four $(66 \%)$ were responders, one $(16 \%)$ was a non-responder. Among the nine patients younger than 30 years, four $(44 \%)$ were responders and four $(44 \%)$ were non-responders (NS).

Gender: There were 11 males $(46 \%)$ and 13 females $(54 \%)$ in the non-responder group compared to 13 males $(52 \%)$ and 12 females $(48 \%)$ in the responder group (NS).

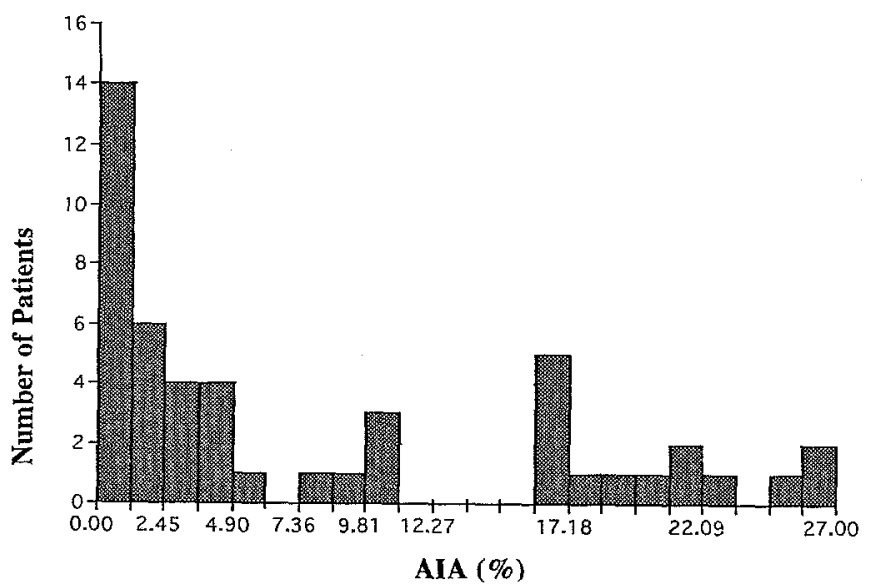

Fig. 1. Mean AIA levels in the second year after implantation. Results are expressed as number of patients per range of AIA level (\%)

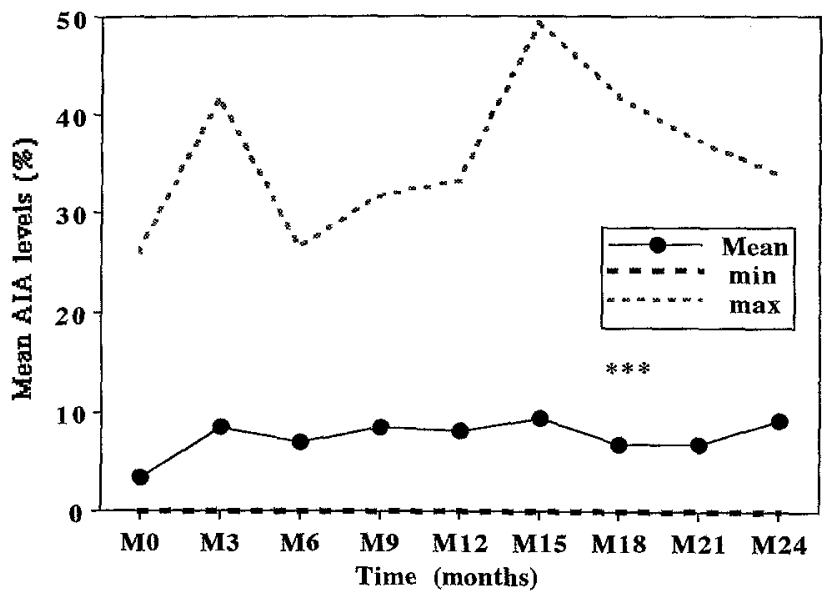

Fig. 2. Evolution of mean AIA levels in Group I after implantation and evolution of lower and higher titres

Results are expressed as mean (\%)

$* * * p<0.001$

Diabetes duration: Mean diabetes duration was similar among responders ( $18.7 \pm 1.6$ years) and non-responders: (15.4 \pm 1.5 years); for the four patients with diabetes duration of less than 5 years, one was a responder, three were non-responders, for the nine patients with diabetes duration longer than 25 years, four were in the non-responder and three were in the responder group (NS).

Insulin therapy prior to implantation. In Group I, only one patient had less than 2 years of insulin treatment, he was treated with multiple injections (MI), his preimplantation AIA level was $0 \%$ and he became a responder. Five control subjects were treated for less than 2 years; they were treated with MI, their AIA levels were respectively: $0,0,0,0$ and $3.5 \%$. Nineteen patients were treated with regular insulin only (continuous subcutaneous insulin infusion (CSII)) and 43 patients used regular and intermediate insulin. 
Table 2. Genetic characteristics of the two groups of patients

\begin{tabular}{lrr}
\hline Alleles & Non-responders & Responders \\
\hline DR3 & 10 & 14 \\
No DR3 & 9 & 9 \\
DR4 & 12 & 17 \\
No DR4 & 7 & 5 \\
C4Aq0 & 4 & 6 \\
No C4Aq0 & 10 & 10 \\
\hline
\end{tabular}

AIA levels prior to implantation were $4.81 \%(0$ $18 \%$ ) for continuous subcutaneous insulin infusion (CSII) patients and $2.77 \%(0-18.5 \%)$ for M I patients (NS). All control subjects except one whose AIA titre was $0 \%$, were M I. All our CSII patients had been on porcine insulin and changed to human insulin 6 to 3 months prior to implant. One year after implant their AIA levels were slightly higher than the $\mathrm{M}$ I patients: $10.43 \%(0-49 \%)$ vs $7.30 \%(0-42 \%)$ (NS).

Genetic background. HLA typing for all patients and complement factor nul alleles for $\mathrm{C} 4$ for the first 39 subjects are shown in Table 2.

Insulin concentration. AIA levels increased in the same way for the two insulin concentrations used in the pumps from $3.39 \%$ (U 100) vs $3.40 \%$ (U 400) to $9.43 \%$ (U 100$)$ vs $7.81 \%$ (U 400) after 3 months. They remained comparable until the end of the study (9.35\% U 100 vs $8.93 \%$ U 400). There was no difference in the ratio non-responder/responder in the group of patients treated with U 400 (36\% nonresponder $-38 \%$ responder) or U 100 (45\% nonresponder $-45 \%$ responder).

AIA level before implantation. There is a significant correlation $(r=0.63 p<0.0005)$ between the AIA levels prior to implantation and the maximum peak reached during the study, a higher level of AIA before implantation was also found in the responder group $(p<0.001)$.

Consequences of metabolic control. Metabolic control improved during the study in the whole population, as well as in the responder and non-responder groups if analysed separately. Comparative data concerning both groups are summarized in Figures 3 to 7. The groups are comparable at time 0 , but metabolic control became statistically better in the non-responder group than in the responder group concerning mean blood glucose $(p<0.05)$, and postprandial blood glucose $(p<0.01)$. The same trend in improvement was observed for $\mathrm{HbA}_{1 \mathrm{C}}$, glycaemic fluctuation as shown by the $24 \mathrm{~h}$ standard deviation $(p=0.07)$. The number of low blood glucose values per month was also lower in the non-responder group. The frequency of severe hypoglycaemic episodes (as defined by the late criteria of the DCCT study [13]) dra-

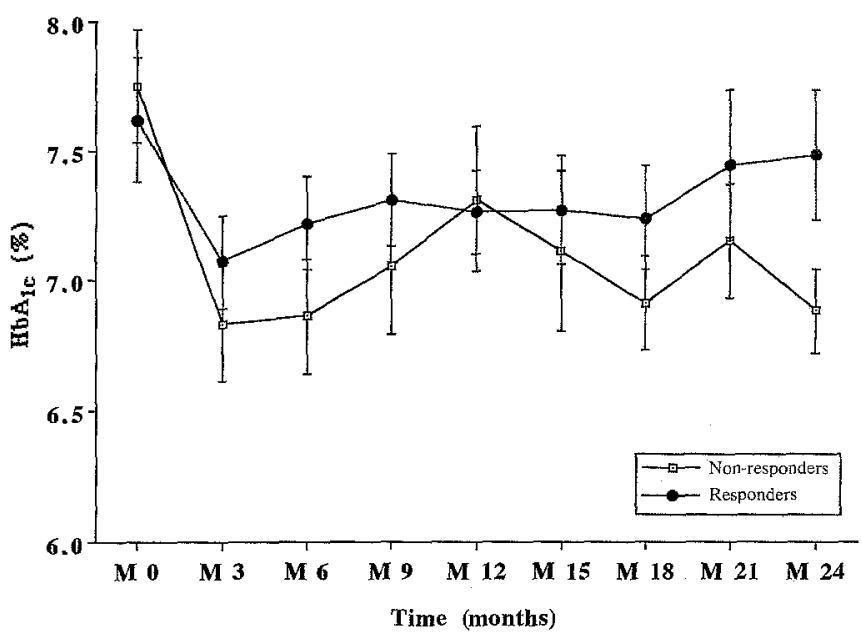

Fig. 3. Evolution of mean $\mathrm{HbA}_{1 \mathfrak{c}}$ levels in non-AIA forming group (non-responders) compared to AIA forming group (responders) after implantation

Results are expressed as mean $\mathrm{SEM} \pm(\%)$

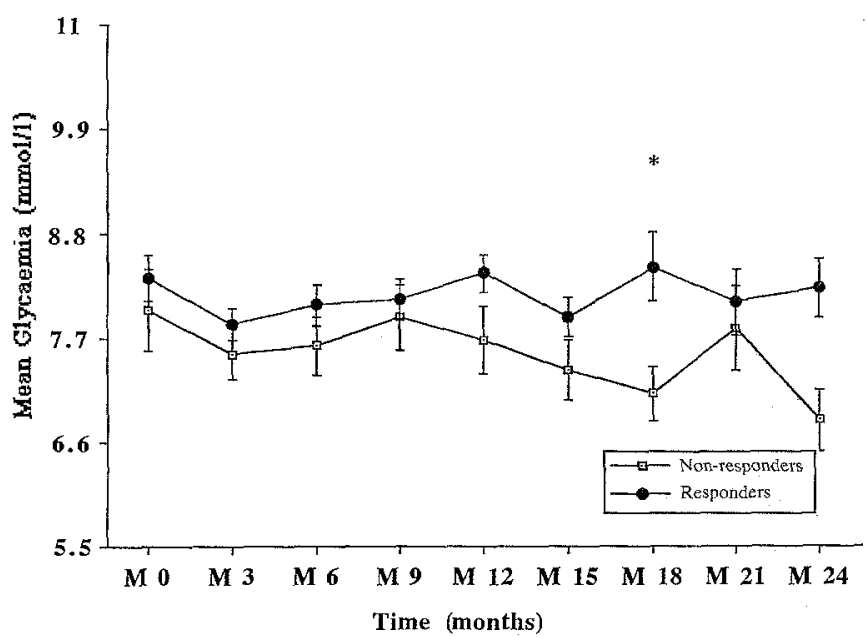

Fig.4. Evolution of mean blood glucose levels in non-AIA forming group (non-responders) compared to AIA forming group (responders) after implantation

Results are expressed as mean \pm SEM (mmol/l)

$* p \leq 0.05$ vs non-responders

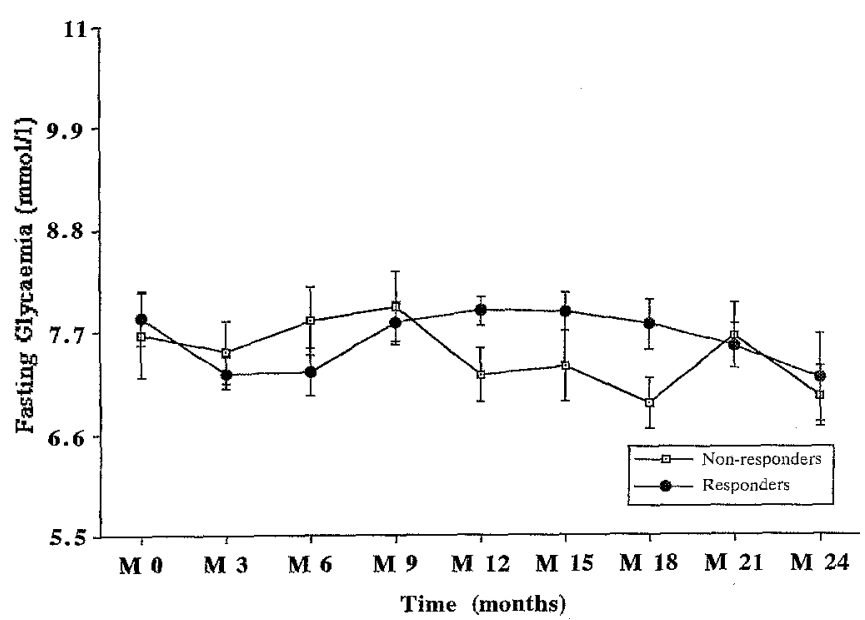

Fig. 5. Evolution of fasting glycaemia in non-AIA forming group (non-responders) compared to AIA forming group (responders) after implantation

Results are expressed as mean \pm SEM 
Table 3. Severe hypoglycaemic episode evolution after implantation

\begin{tabular}{lccl}
\hline & $\begin{array}{l}\text { Non-re- } \\
\text { sponders }\end{array}$ & $\begin{array}{l}\text { Re- } \\
\text { sponders }\end{array}$ & $\begin{array}{l}\text { Non-re- } \\
\text { sponders and } \\
\text { responders }\end{array}$ \\
\hline $\begin{array}{l}\text { Before implantation } \\
\text { (per 100 Patient x years) }\end{array}$ & 138.9 & 150.0 & 144.6 \\
$\begin{array}{l}\text { After implantation } \\
\text { (per 100 Patient x years) }\end{array}$ & 21.7 & 2.7 & 10.8 \\
\hline
\end{tabular}

Calculated for a mean of 1.4 years per patient compared to pre-implantation (calculated during 2 years for each patient) in non-AIA forming group (non-responders) and AIA forming group (responders). Expressed as numbers per 100 patient years

matically decreased during pump therapy for all patients as shown in Table 3. No statistically significant difference in insulin requirements was found between the responder and non-responder groups during the entire course of the study. Insulin requirements decreased significantly for non-responder patients $(p<0.01)$. In the responder group, after a significant decrease during the first 9 months $(p<0.01)$, insulin requirements increased again to pre-implantation levels and then remained stable until the end of the study (Fig. 8).

Impact on catheter function. Frequency of catheter obstruction was similar in the two groups, $20 \%$ $(n=4)$ in the non-responder group and 16\% $(n=3)$ in the responder group, the small number of catheter obstructions in the two groups does not allow any statistically significant difference $(r=3)$.

Clinical consequences: two case reports. The clinical relevance of very high AIA titres remains to be assessed, since in some cases it may lead to interruption of therapy. Two of our patients, one male and one female (both HLA DR4) whose AIA titres were very high, up to $40 \%$, suffered from clinical symptoms similar to the "insulin autoimmune syndrome" [14] such as a dramatic nocturnal fall in blood glucose (5.5 to $11 \mathrm{mmol} / 1$ despite a complete pump stop for 6 to $8 \mathrm{~h}$ ) and a very severe insulin resistance during the day.

One of the patients often had massive ketonuria during the day with a basal rate of $3 \mathrm{U} / \mathrm{h}$ and blood glucose around $11 \mathrm{mmol} / \mathrm{l}$. $\mathrm{HbA}_{1 \mathrm{c}}$ increased from $6.9 \%$ at the beginning of the symptoms (AIA of $20 \%$ ) to $10.5 \%$ at the end of pump therapy (AIA of $25.7 \%$ ). Free insulin levels increased at night time from an average of $15 \mu \mathrm{U} / \mathrm{ml}$ during the day to $77 \mu \mathrm{U} / \mathrm{ml}$ at 24.00 hours and $39 \mu \mathrm{U} / \mathrm{ml}$ at 04.00 hours. At the same time total insulin levels were over $192000 \mu \mathrm{U} / \mathrm{ml}$. A laparoscopy was performed, the catheter was not encapsulated and the peritoneum was free of adhesions, the pump was changed and thus

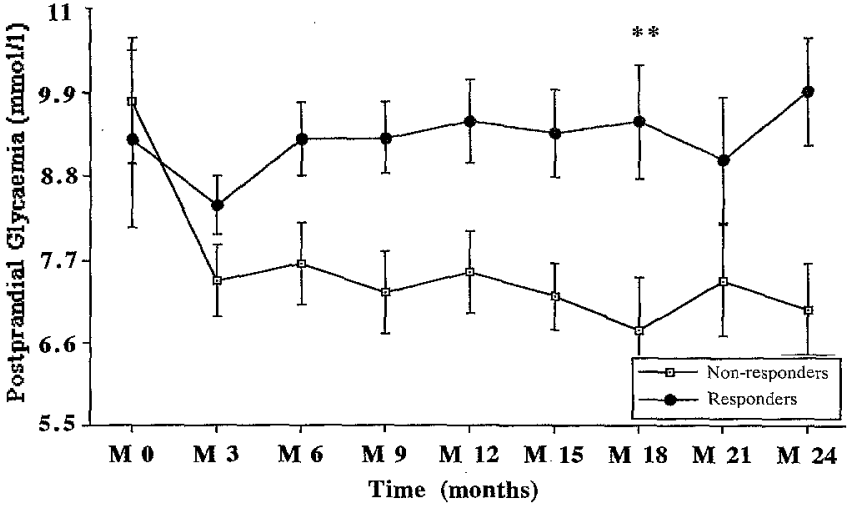

Fig. 6. Evolution of postprandial glycaemia in non-AIA forming group (non-responders) compared to AIA forming group (responders) after implantation

Results are expressed as mean \pm SEM

$* * p \leq 0.05$ vs non-responders

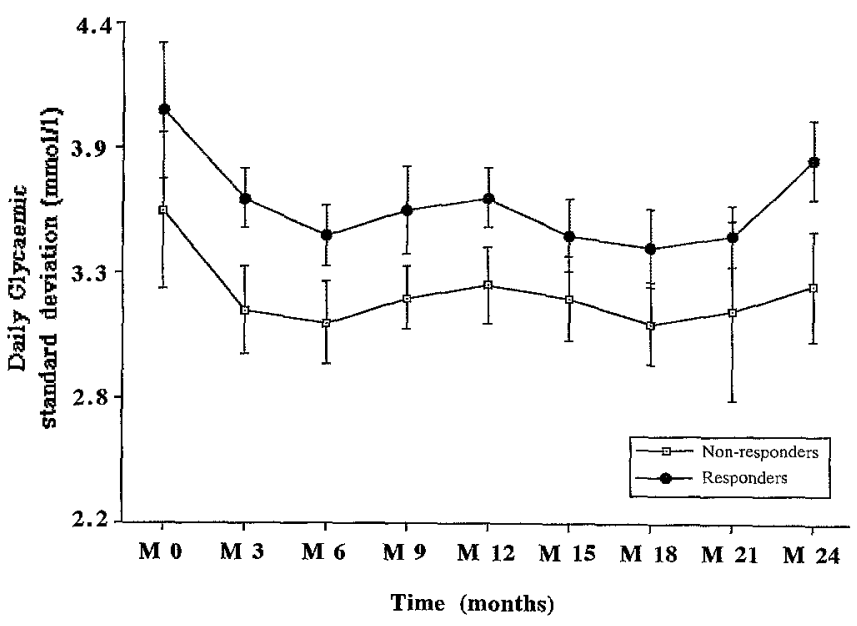

Fig. 7. Evolution of daily glycaemic standard deviation in nonAIA forming group (non-responders) compared to AIA forming group (responders) after implantation

Results are expressed as mean \pm SEM

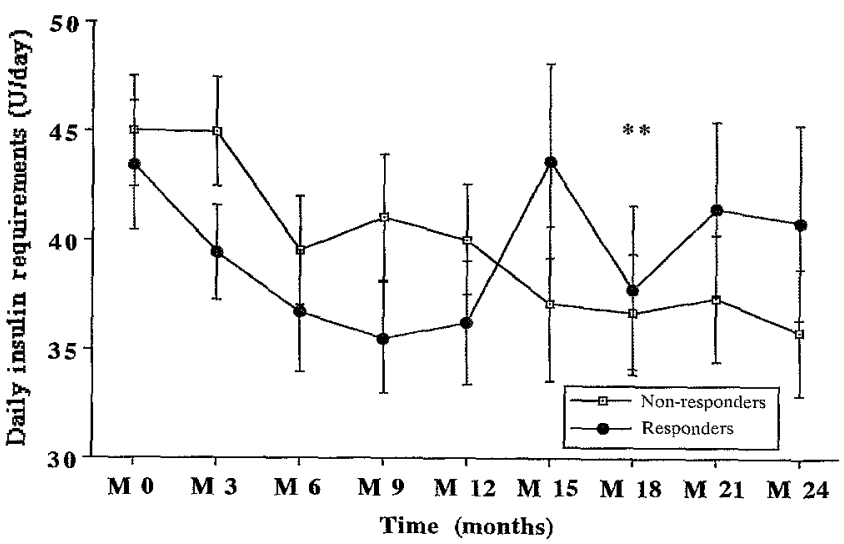

Fig. 8. Evolution of daily insulin requirements in non-AIA forming group (non-responders) compared to AIA forming group (responders) after implantation

Results are expressed as mean \pm SEM

** $p \leq 0.01$ vs the first 2 months after implantation 
the insulin concentration changed from U 100 to U 400. Metabolic results were slightly improved for about 1 month, in particular the insulin resistance symptoms (disappearance of ketonuria), then all the symptoms reappeared. The patient was changed from i.p. infusion to a porcine insulin subcutaneous infusion; the symptoms remained stable for about 9 months and are now improving with a stabilization of nighttime blood glucose levels and a decrease in daytime insulin requirements. It is very difficult to definitely link these types of symptoms to a high AIA level, even though the patient's AIA levels tended to decrease slightly after changing to porcine subcutaneous therapy, AIA titres were at $28.7 \%$ when i.p. insulin was resumed, $30 \%$ after 4 months of subcutaneous therapy and $17.8 \%$ at the improvement stage. To detect an eventual difference between the types of AIA observed in these patients compared to the others, a Scatchard technique [15] was performed on four other patients presenting very high AIA levels (over $30 \%$ ) and severe, light or no clinical symptoms. There was no striking difference between the AIA constant of association or the number of high affinity sites.

\section{Discussion}

Our data confirm the positive effect of i.p. insulin infusion on metabolic control and particularly on diabetes stability, with a drastic reduction of severe hypoglycaemic episodes associated with an improvement of diabetes control [2]; this was a positive aspect considering the results of the DCCT study [13]. In our study the frequency of severe hypoglycaemia decreased in both groups (non-responder and responder) in the same way, showing that high AIA levels do not seem to increase the risk of severe hypoglycaemic episodes. Even if the pre-implantation data are retrospective, based on the patients, files and questionnaires from the 2 years prior to implant, the post-implantation data are prospective and the huge discrepancy between the frequency of events remains interesting and close to other published results [2].

Our data show that i.p. insulin leads to a high immunogenic response to insulin and this has been confirmed by others $[5,6,8,9]$. Concerning their immune response towards insulin, our patients behave as normal diabetic patients, since their AIA levels prior to implantation were comparable to the AIA levels of the unselected IDDM patients seen in our clinic at the same time. Therefore, the increase in AIA levels observed in our study is probably specific to the mode of therapy.

AIA are very hard to characterize and to quantify, different assays may assess different types of antibodies whose properties and functions are not well understood. Differences may exist between the meth- ods used and between assays. No "gold standard" method is yet available. Radioimmunoassay dosages measure high affinity antibodies, and ELISA assay measures more specifically medium to low affinity AIA [16]. This adds to the difficulty of comparing titres between different studies.

AIA may fluctuate over time in a given patient as in a classic immune response with first, the formation of anti-insulin IgG followed by anti-IgG antibodies [17]. These fluctuations should be considered since most of the previous studies report only one or two titre values during the follow-up.

To minimize these difficulties, we evaluated the potential of each patient to achieve a high immune response. Therefore, we decided to look at the population AIA titres repartition once the immune response was achieved (after 12 months). Mean AIA levels in the non-responder group remain in the range considered as negative for the assay $(n<2 \%)$ : $0.53 \%$ prior to implantation, $0.77 \%$ after 24 months.

In order not to characterize our patients wrongly (classify a responder as a non-responder) we studied the AIA titres every 3 months and we checked that no new patient reached an AIA titre of $15 \%$ after 18 months. Our data clearly show that only half of the patients had a significant increase in AIA titres; thus, there must be other factors involved.

Different factors described in the literature as being involved in AIA formation were studied. Our preliminary data [6] and those from other authors $[18,19]$ have found some genetic factors (HLA DR3 or complement factor allele $\mathrm{C} 4 \mathrm{AQ} 0$ ) to be related to low AIA levels. This is not confirmed here, where none of the genetic factors seem to statistically influence the AIA titres, despite a tendency for the DR4 population to be part of the responder group. The small number of patients in each subgroup may be the cause of the absence of statistical significance. Older subjects seem more likely to become responders, as would be expected [20]. The other factors studied, i. e. diabetes duration and gender, do not seem to influence AIA formation.

Regular insulin is usually less immunogenetic than intermediate insulin, but our CSII patients had recently been treated with porcine insulin which may explain the slightly elevated AIA titres. Most of our patients had been insulin treated for a long time. Among the few recently treated, the very low AIA titres do not confirm the hypothesis of a high rise in AIA titres at the beginning of any subcutaneous human insulin therapy.

The AIA value of patients prior to implantation is related to the evolution of AIA levels, but is not predictive since $25 \%$ of the patients with an AIA titre of $0 \%$ prior to implantation became responders.

The route of insulin administration may influence the immunogenicity of insulin since some authors have described a sustained decrease in AIA titres 
after 6 and 12 months of intravenous insulin therapy using implantable devices [21] in non-insulin-dependent diabetic patients. A particular immunogenicity may be related to the peritoneal route since mice produce higher quantities of AIA in their serum after an i.p. insulin injection than after a subcutaneous injection [22]. Peritoneal B lymphocytes have also been described in mice as Ly 1 B lymphocytes, which are very sensitive and more reactive to immune stimulation than other classes of lymphocytes found in other tissues [23].

The insulin used in this study is a semi-synthetic human purified insulin and thus should show very little immunogenicity [24-26]. In this study neither the difference in insulin concentration nor the type of device used had any consequence in the evolution of AIA titres.

The Genapol used to stabilize the insulin may be one of the factors to consider, by acting as a carrier. Some studies concerning this insulin [27] have reported that $5 \%$ of the insulin infused could be modified before reaching the body. The chemical nature of the derivative is not yet known but possesses full biological potency. The fact that it does not lead to increased AIA titres in pigs compared to standard human insulin does not exclude a role for this denatured product in our observations.

It has been reported that $95 \%$ of native insulin is found in the residual insulin collected from the pump reservoir during each refill, a small amount consists of desamidoinsulin (HPLC) which does not seem to be particularly antigenic in rabbits but may be immunogenetic in humans [28]. We found no data in the literature concerning the eventual immunogenicity of the high molecular weight component found by analysing the residual insulin by size exclusion chromatography [29]. Insulin aggregates have been shown to induce specific antibodies [30] and may be involved in the immune reaction observed.

As already reported, AIA do influence metabolic control [31-35] particularly postprandial blood glucose. Some authors [9] found no direct correlation between the AIA levels and glycaemic control, nor did we. That might be explained by the high number of other parameters involved in the diabetic control at a given time (anxiety, diet, transient illness, technical problems). These parameters might have a greater influence on metabolic control, the influence of AIA titres remaining moderate.

Insulin requirements decreased at first in both responder and non-responder groups, due perhaps to improvement in diabetic control, and then increased again in the responder group after 9 months, closely following the AIA titres evolution. Nevertheless, insulin requirements remained comparable in both groups during the entire study.

Some "morning lows" have also been described [5] with the observation of clinical symptoms as soon as the AIA titres are over $20 \mu \mathrm{U} / \mathrm{ml}$. In our study clinical symptoms did not strictly correlate with the AIA titres and remained the same despite the titres fluctuations, four patients reached the same or higher levels of AIA $(40 \%)$ without any clinical symptoms. Since the RIA technique is known to select high avidity AIA, we could have missed some low or medium affinity AIA which could well be involved in these "morning lows", the low or medium affinity sites being linked more directly to the insulin bioavailability [36].

The i.p. insulin infusion by means of implantable pumps significantly improves diabetic control and stability, with a dramatic decrease in severe hypoglycaemic episodes. Our data clearly demonstrate that this mode of therapy is immunogenetic, but that the elevation of AIA titres affects only half of the treated patients and has moderately deleterious effects on metabolic control and diabetic stability. A possible implication in rare cases of severely destabilized diabetes is suggested. Further studies are needed in order to isolate the factors and causes involved in these very rare clinical symptoms, in order to characterize these types of patients as "at-risk patients" who should not be given implants or the peritoneal route should be avoided as long as no simple therapy is found. Sulphated insulin has been tried in a few cases of insulin resistance but does not seem to be very efficient [37], and steroids are not easy to use in diabetic patients. Recent studies have shown that different IgG sub-classes are triggered during insulin therapy in man [38]; since the function of these subclasses is different it would be interesting to compare these different fractions in the patients suffering from the "morning low syndrome" and in other patients with high antibody levels and no symptoms.

Acknowledgements. The authors thank Ms A. Lazarus for secretarial support, Ms L. Hoekstra for her advice on English language, and P.Meyer M.D. (University Louis Pasteur, Department of Statistics) for help concerning the statistical analysis. The Centre Européen d'Etude du Diabète is thanked for the technical and financial support.

\section{References}

1. Saudek CD, Selam JL, Pitt THA et al. (1989) A preliminary trial of the programmable implantable medication system for insulin delivery. N Engl J Med 321: 574-579

2. Broussolle C, Jeandidier N, Hanaire-Broutin H (1994) French multicentre experience of implantable insulin pumps. Lancet 343: 514-515

3. Selam JL, Raccah D, Jeandidier N, Lozano J, Waxman K, Charles MA (1992) Randomized comparison of metabolic control achieved by intraperitoneal insulin infusion with implantable pumps versus intensive subcutaneous insulin therapy in type I diabetic patients. Diabetes Care 15: $53-58$

4. Olsen CL, Liu G, Iravani M, Nguyen S et al. (1993) Long term safety and efficacy of programmable implantable insulin delivery systems. Int J Artif Organs 16: 847-854 
5. Olsen CL, Chan E, Turner D et al. (1994) Insulin antibody responses after long-term intraperitoneal insulin administration via implantable programmable insulin delivery systems. Diabetes Care 17: 169-176

6. Boivin S, Jeandidier N, Buckenmeyer V, Sapin R, Derdour H, Pinget M (1994) Effect of immunogenicity of long term continuous peritoneal insulin infusion (CPII) on metabolic parameters in type I diabetic patients. Horm Metab Res 26: A7 (Abstract)

7. Bousquet-Rouaud R, Chante MA, Orsetto A, Mirouze J (1990) Increase in anti-insulin antibody titer during continuous peritoneal insulin infusion. Artficial Organs 14 [Suppl 3]: 241-244

8. Charles MA, Dunn F, Nathan D, Scavini M (1993) Insulin antibody levels after 2-3 years of intraperitoneal or intravenous insulin using implantable insulin delivery. Diabetologia 36 [Suppl 1]: A161 (Abstract)

9. Lassmann-Vague V, Belicar P, Raccah D, Vialettes B, Vague $\mathrm{Ph}$ (1993) Immunogenicity of long term intra-peritoneal insulin infusion and relation to metabolic control. Diabetologia 36 [Suppl 1]: A38 (Abstract)

10. Selam JL, Charles MA (1990) Devices for insulin administration. Diabetes Care 13: 955-959

11. Delahunty T (1990) Convenient screening for hemoglobin variants by using the Diamat HPLC system Clin Chem 36: 903-905

12. Vartdal F, Gaudernack G, Funderud S et al. (1986) HLA class I and class II typing using cells positively selected from blood by immunomagnetic isolation: a fast and reliable technique. Tissue Antigens 28: 301

13. The DCCT Study Group (1993) The effect of Intensive treatment of Diabetes on the Development and progression of long term complications in insulin dependent diabetes mellitus. N Engl J Med 329 (14): 977-986

14. Ushigata Y, Kuwata S et al. (1992) Strong association of insulin autoimmune syndrome with HLA DR4. Lancet 339 394

15. Scatchard G (1949) The attractions of proteins for small molecules and ions. Ann New York Acad. Sci. 51: 660-672

16. Sodoyez JC, Koch M, Sodoyez-Goffaux F (1991) Anticorps anti-insuline: méthodologie et implications cliniques. Diabète et Métabolisme 17: 255-269

17. Roitt I (1989) In: Pradel (ed) Immunologie. Editions Pradel no first name Initials. City Paris 75001 France. Publisher Jouve (18 rue Saint Denis 75001 Paris) p 122

18. Kahn CR, Mann D, Rosenthal AS, Galloway JA, Johnson AH, Mendell N (1982) The immune response to insulin in man. Interaction of HLA alloantigens and the development of the immune response. Diabetes 31: 716-723

19. Reeves WG, Barr D, Douglas CA et al. (1984) Factors governing the human immune response to injected insulin. Diabetologia 26: 266-271

20. Andersen O (1972) Insulin antibody formation. The influence of age, sex, infections, insulin dosage and regulation of diabetes. Acta Endocrinol 71: 126-140

21. Chute EP, Rupp WM, Barbosa JJ et al. (1985) Anti-insulin antibody titers after one year of intravenous insulin infusion by implantable pump. Surgical Forum 36: 189-191

22. Overkamp D, Mohammed Ali S, Cartledge C, Landon J (1988) Production of polyclonal antibodies in ascitic fluid of mice: technique and appolications. J Immunoassay 9 (1): $51-58$

23. Lalor P, Mora G (1990) The peritoneal Ly (CD5) B cell repertoire is unique among murine B cell repertoire. Eur J Immunol 20: 485-492

24. Fineberg SE, Galloway JA, Fineberg NS, Goldman J (1983) Effects of species of origin, purification levels, and formulation on insulin immunogenicity. Diabetes 32: 592-599

25. Heding LG, Larsson Y, Ludvigsson J (1980) The immunogenicity of insulin preparations: antibody levels before and after transfer to highly purified porcine insulin. Diabetologia 19: 511-515

26. Kurtz AB, Matthews JA, Mustaffa BE, Daggett PR, Nabarro JDN (1980) Decrease of antibodies to insulin, proinsulin and contaminating hormones after changing treatment from conventional beef to purified pork insulin. Diabetologia 18: 147-150

27. Grau U (1985) Chemical stability of insulin in a delivery system environment. Diabetologia 28: 458-463

28. Kasama T, Iwata $Y$ et al. (1981) Antigenicity of desamidoinsulin and monocomponent insulin. Diabetologia 21: 6569

29. Grau U, Saudek C (1987) Stable insulin preparation for implanted insulin pumps. Diabetes 36: 1453-1459

30. Robbins DC, Cooper SM, Fineberg SE, Mead PM (1987) Antibodies to covalent aggregates of insulin in blood of insulin-using diabetic patients. Diabetes 36: 838-841

31. Chantelau E, Sonnenberg GE, Heding LG, Berger M (1984) Impaired metabolic response to regular insulin in the presence of high level of circulating insulin-binding immunoglobulin G. Diabetes Care 7: 403

32. Van Haeften TW, Heiling VJ, Gerich JE (1987) Adverse effects of insulin antibodies on postprandial plasma glucose and insulin profiles in diabetic patients without immune insulin resistance. Implications for intensive insulin regimens. Diabetes 36: 305-309

33. Van Haeften TW (1989) Clinical significance of insulin antibodies in insulin-treated diabetic patients. Diabetes Care 12: $641-648$

34. Waldhausl W, Bratusch-Marrain P, Kruse V, Jensen I, Nowotny P, Vierhapper H (1985) Effect of insulin antibodies on insulin pharmacokinetics and glucose utilization in insulindependent diabetic patients. Diabetes 34: 166-173

35. De Pirro R, Fusco A, Spallone L, Magnatta R, Lauro R (1980) Insulin antibodies prevent insulin-receptor interactions. Diabetologia 19: 118-122

36. Koch M, Sodoyez JC, Sodoyez-Goffaux F, Dozio N, Di Silvio LS, Kurtz AB (1989) Is quantitative assessment of insulin-antibodies and autoantibodies feasible? Diabetologia 32: $774-778$

37. Davidson JK, Fineberg SE, De Meyts P, Fineberg N, Galloway JA (1992) Immunological and metabolic responses of patients with history of antibody-induced beef insulin resistance to treatment with beef, pork, human, and sulfated beef insulin. Diabetes Care 15: 702-704

38. Koch M, François-Gerard C, Sodoyez-Goffaux F, Sodoyez JC (1986) Semi-quantitative assessment of anti-insulin total IgG subclasses in insulin immunised patients using a highly sensitive immunochemical micromethod. Diabetologia 29: 720-726 